\title{
SIMULATION STUDIES OF BEAM DYNAMICS IN 50 MEV LINEAR ACCELERATOR WITH LASER-PLASMA ELECTRON GUN
}

\author{
Sergey M. Polozov \\ National Research Nuclear University- \\ Moscow Engineering Physics Institute \\ Russia \\ SMPolozov@mephi.ru
}

\author{
Vladimir I. Rashchikov \\ National Research Nuclear University- \\ Moscow Engineering Physics Institute \\ Russia \\ VIRashchikov@mephi.ru
}

Article history:

Received 17.11.2021, Accepted 20.12.2021

\begin{abstract}
Conventionally, electron guns with thermionic cathodes or field-emission cathodes are used for research or technological linear accelerators. RF-photoguns are used to provide the short electron bunches which could be used for FEL's of compact research facilities to generate monochromatic photons. Low energy of emitted electrons is the key problem for photoguns due to high influence of Coulomb field and difficulties with the first accelerating cell simulation and construction. Contrary, plasma sources, based on the laser-plasma wakefield acceleration, have very high acceleration gradient but rather broad energy spectrum compare with conventional thermoguns or field-emission guns. The beam dynamics in the linear accelerator combines the laser-plasma electron source and conventional RF linear accelerator is discussed in this paper. Method to capture and re-accelerate the short picosecond bunch with extremely broad energy spread (up to $50 \%$ ) is presented. Numerical simulation shows that such bunches can be accelerated in RF linear accelerator to the energy of $50 \mathrm{MeV}$ with output energy spread not higher than $1 \%$.
\end{abstract}

\section{Key words}

Laser-plasma wakefield acceleration, beam dynamics, longitudinal and transverse motion stability.

\section{Introduction}

The high brightness electron sources are usually based on photoguns. Photojnjectors should solve a number of tasks which sufficiently differs by the bunch parameters and operation regimes. First class of photoguns are used for generation of high-intensity bunches for X-FEL's such as European-XFEL or LSLS. In such facilities bunches with charge $\sim 1 \mathrm{nC}$, bunch length $\sim 1 \mathrm{ps}$, transverse emittance less than $10 \mathrm{~mm}$ mrad and repetition rate up to $1000 \mathrm{~Hz}$ should be generated. The second class of photoguns is used for Compact-XFEL's and needs lower bunch charges (not higher than $100 \mathrm{pC} /$ bunch) but the bunch length should be also shorter: 100-300 fs with the trend to tens of fs. The third class requires bunches with charges $\sim 100 \mathrm{pC}$ but with high repetition rate of $10-100 \mathrm{kHz}$. The bunch charge of a photogun is limited by quantum efficiency of cathode and non-linear effect at the near-cathode region. The photocathode life-time is also limited and currently amounts not higher than few hundreds of hours for semiconductor (Cs2Te, etc.) cathodes. Moreover the life-time is also limited by the total emitted charge.

One possible way to solve the problems with higher bunch charges, shorter bunch lengths and longer lifetimes is to use the plasma electron sources. As known plasma wakefield acceleration (PWA) was proposed many years ago by Ya.B. Feinberg [Feinberg, 1959] to solve the problem of accelerating field limitation in conventional RF structures. Two different ways to form the plasma channel were proposed: bunch PWA (see for example [Hogan, 2005]) and laser PWA [Tajima, 1979]. It is necessary to use GeV-energy electron bunches to realize high efficiency bunch PWA and this technique is successfully realized experimentally. The second way is much more popular but the results are contradictory: simulations and experiments demonstrates very high rate of the energy gain (up to hundreds $\mathrm{GV} / \mathrm{m}$ ) but the output energy spectrum is broad (not less than $10 \%$ for energies of $0.1-1 \mathrm{GeV}$ ) and the capturing coefficient is very low (not higher than 1-3\%). Few ways to decrease the energy spectrum which is too broad for further acceleration in real applications: ramping, ponderomotive injection, modulation of plasma density, non-linearities in plasma 
[Bulanov, 1998; Umstadter, 1996; Polozov, 2013; Polozov, 2018]. Bunch spectrum can be decreased by the factor 3-4 by means of these methods, but the spectrum value of $\sim 3 \%$ for the energy $\sim 1 \mathrm{GeV}$ is ten times higher than for standing wave RF linear accelerator.

As mentioned above we will discuss the combination of PWA electron source with output energy of few hundreds of $\mathrm{keV}$ and standing wave RF accelerating structure to generate the short ( $1 \mathrm{ps}$ or less) electron bunches with the charge of $100 \mathrm{pC}$, variable output energy of 20$50 \mathrm{MeV}$ and narrow energy spectrum less than $1 \%$. It is proposed to use the cluster plasma jet with laser-plasma acceleration for electron bunch generation with energy above $1 \mathrm{MeV}$, length less than $1 \mathrm{ps}$ and charge of 50200 pC [Shkurinov, 2017; Shkurinov, 2021; Semenov, 2019].

\section{Beam Dynamics Simulations}

It was proposed to use RF linear accelerator consists of two sections. Both sections will be based on the biperiodic accelerating structure (BAS) operating on the standing wave. The first section should be used for capturing of the bunch and to made bunch gymnastics to decrease the energy spectrum (call it "matching section"). Such section will includes seven accelerating cells. Two first cells will be bunching cells with phase velocities less than the velocity of the light. For example, the first cell will has phase velocity normalized to the speed of light 0.95 and the second 0.96 for the injection energy 800 $\mathrm{keV}$. Note that the first accelerating cell should be halfcell like that in photoguns (so-called "electrical wall"). Other five accelerating cells will be regular with phase velocity equals 1 and the RF power coupler should be arranged in one of these cells. The length of sections will be $31 \mathrm{~cm}$ and $214 \mathrm{~cm}$ respectively. Both sections operates on the frequency of $2800 \mathrm{MHz}$.

The beam dynamics simulation in the proposed linear accelerator was done by using BEAMDULAC-BL code which was developed at MEPhI to simulate dynamics taking into account both quasistatic component of the own space charge field and beam loading self-consistently [Polozov, 2010; Bondarenko, 2013; Rashchikov, 2020; Kluchevskaia, 2020]. It was shown than the bunch generated by cluster plasma electron gun can be efficiently captured by the first section. Simulation was done for low intensity bunches with charge $100 \mathrm{pC} /$ bunch, energy $800 \mathrm{keV}$, transverse emittance $3 \mathrm{~mm}$ mrad, bunch length 1 ps. The accelerating field amplitude of $400 \mathrm{kV} / \mathrm{cm}$ gives the bunch energy 8.3 MeV after first section. Optimal injection phase is equal to $3.4 \mathrm{rad}$. The output energy spectrum will be defined by the parameters of the first accelerating cell as well as by the bunch initial energy spread. Simulations were done for different values of injection spectra from $1 \%$ to $50 \%$ FWHM. The main simulation results are presented in Fig. 1 and Table 1. Phase portraits and en- ergy spectra are shown for injection and after sections, injection parameters are shown by red color, after 1st section by blue, after regular section by black. It is clear that such capturing scheme is efficient and output spectrum can be sufficiently reduced, bunch capturing coefficient is close to $100 \%$ and bunch is prepared for reacceleration in the regular section. The energy spectrum range from $1.8 \%$ to $5.1 \%$ FWHM will be observed for the bunch with the initial spectrum from $1 \%$ to $50 \%$. It should be noted that the capturing process will dramatically depends from the initial bunch length and the injection energy. The first accelerating call length, phase velocity and RF field amplitude should be chosen assuming the averaged value of the electrons velocities inside this cell. The cell parameters should be optimized for each injection velocity. Bunches having the duration longer than 2-3 ps will captured and accelerated with current transmission coefficient less than $100 \%$. The designed structure will be not very flexible for the bunch injection parameters due to.

Following the beam dynamics simulation for regular section was studied. It was shown that we may achieve very narrow energy spectrum for the energy range of 50 $\mathrm{MeV}$. It will varies from 0.1 to $1.1 \%$ FWHM and this result shows that the influence of injection parameters can be decreased by means of accurate tuning of matching section and injection phases (optimal phase shift between matching and regular sections will be defined as 1.6-1.7 rad). The simulated values of the output spectra vs. injection one were also summarized in the Table 1 and phase portraits and spectra are shown in Fig. 1.

Table 1. Bunch energy spread (\%) after matching and regular sections vs. injection one.

\begin{tabular}{c|c|c}
\hline Injection & Buncher & Regular \\
\hline \hline 1.0 & 1.8 & 0.084 \\
10.0 & 2.1 & 0.224 \\
30.0 & 3.1 & 0.419 \\
50.0 & 5.1 & 1.095 \\
\hline
\end{tabular}

The dependence of output energy from the accelerating field amplitude was studied. It was proposed that the RF field amplitude will constant $(400 \mathrm{kV} / \mathrm{cm})$ in the matching section and will be varied from $100 \mathrm{kV} / \mathrm{cm}$ to $400 \mathrm{kV} / \mathrm{cm}$ in the regular one. Such field amplitude variation allows to have the output energy after second section from $20 \mathrm{MeV}$ to $53 \mathrm{MeV}$. This dependence is presented in Table 2, phase portraits and energy spectra are shown in Fig. 2. Note that the dependence of the spectrum vs. output energy is close to classical trend for RF linear accelerators $\Delta \gamma / \gamma \sim 1 / \sqrt{\gamma}$. 
$\Delta \gamma / \gamma=1 \%$

Injection
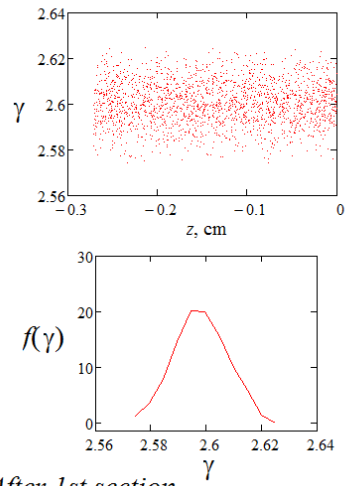

After 1st section

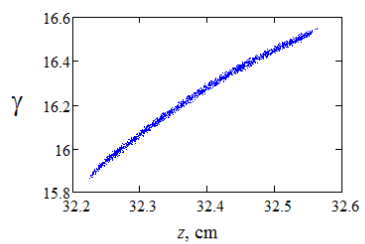

$f(\gamma)$

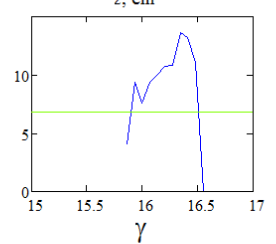

After reg. section
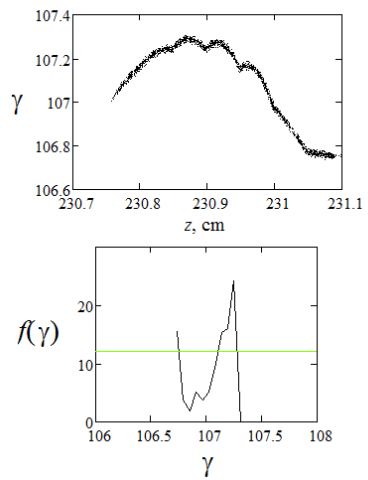

$\Delta \gamma / \gamma=10 \%$

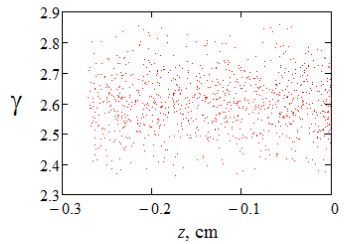

$f(\gamma)$
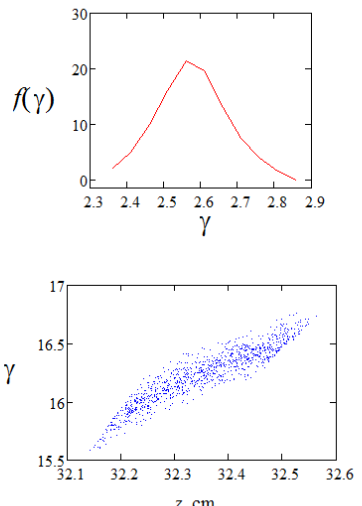

$f(\gamma)$
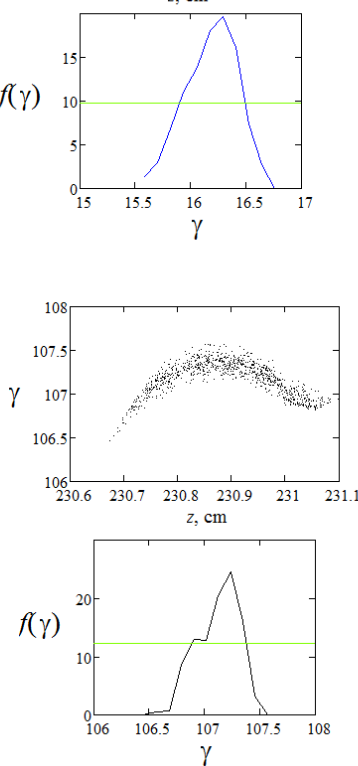

$\Delta \gamma / \gamma=30 \%$

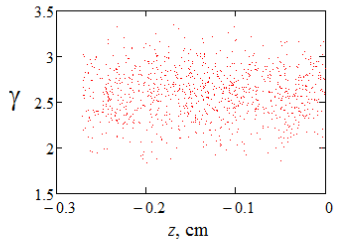

$f(\gamma)$
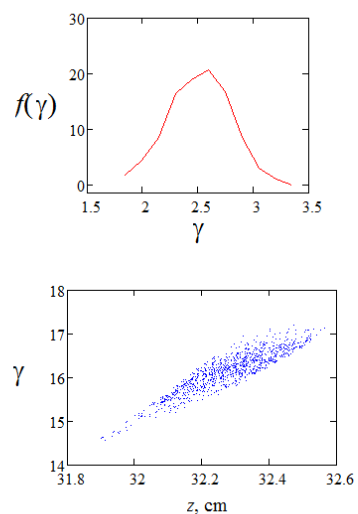

$f(\gamma)$
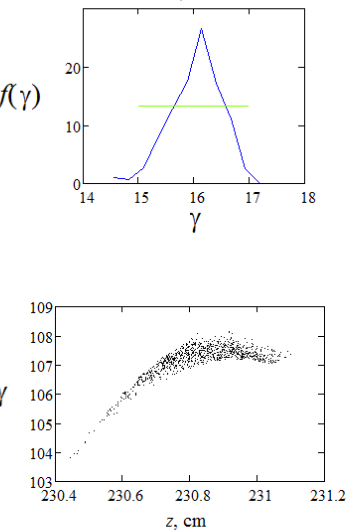

$f(\gamma)$

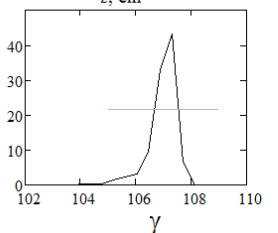

$\Delta \gamma / \gamma=50 \%$

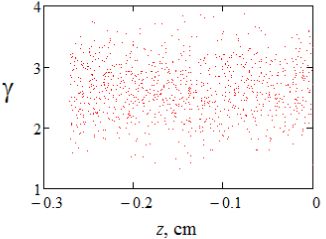

$f(\gamma)$
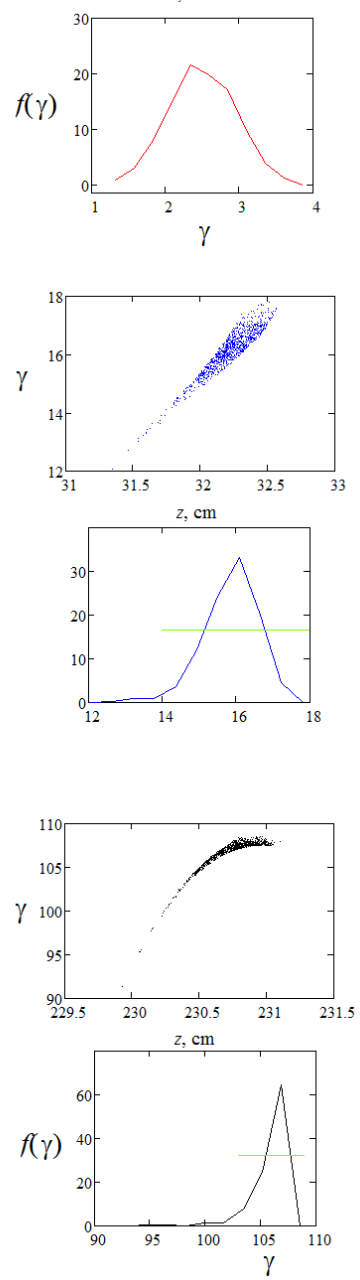

Figure 1. Beam dynamics simulation results for the matching and the regular sections. Phase portraits and energy spectra are shown for injection (by red color), after matching section (by blue) and regular one (by black). 

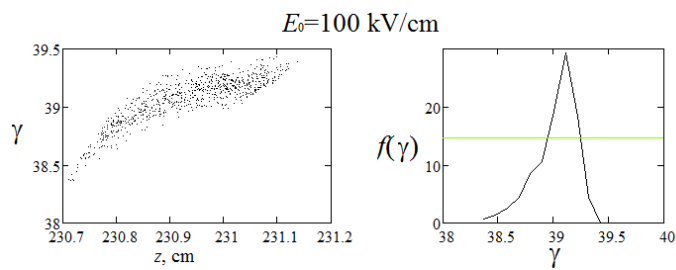

$E_{0}=200 \mathrm{kV} / \mathrm{cm}$
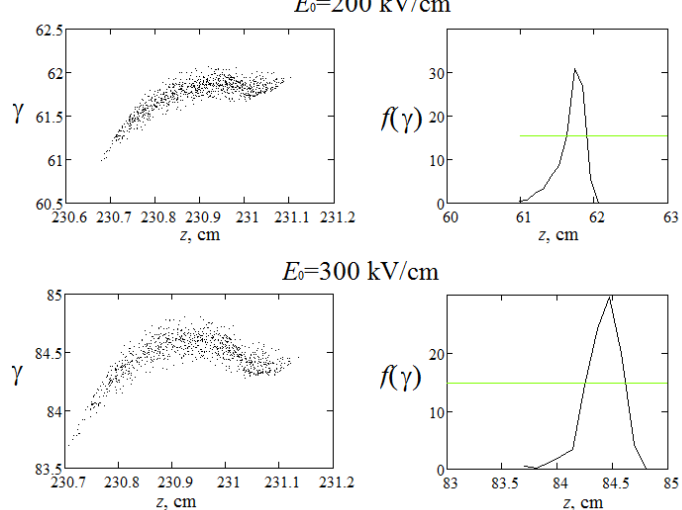

$E_{0}=400 \mathrm{kV} / \mathrm{cm}$
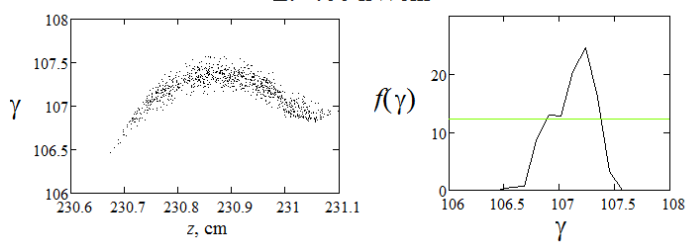

Figure 2. Bunch output phase portraits and energy spread vs. accelerating field amplitude for regular section.

\section{Conclusion}

In this paper we discussed first results of the beam dynamics simulation in RF linear accelerator with injection form the laser-plasma based electron gun. It was proposed to use the cluster plasma source to generate short (less than $1 \mathrm{ps}$ ) electron bunches with energy of few hundreds of $\mathrm{keV}$ and re-accelerate them in conventional RF linear accelerator. Such idea can be used as one of possible compromises between photogun and LPWA: bunches will have narrow energy spread compare with LPWA and the plasma cathode could be considered" perpetual" opposed to short life-time for semiconductor photocathodes. The injection energy will be much more higher for plasma cathode comparatively with photocathode and therefore the influence of Coulomb repulsion can be neglected due to. Simulation shows that the influence of broad energy spectra of injected bunches after plasma electron source can be decreased by means of accurate tuning of matching section and optimal choice of injection phases. The beam energy spectrum can be reduced to $1 \%$ for the energy of $50 \mathrm{MeV}$.

\section{Acknowledgements}

Work financially supported by Ministry of Science and Higher Education of the Russian Federation in framework of Agreement No.075-15-2021-1358 from 12 Oc- tober 2021.

Table 2. Bunch output energy and energy spread vs. accelerating field amplitude for regular section.

\begin{tabular}{c|c|c}
\hline $\begin{array}{c}\text { RF field } \\
\text { amplitude, } \mathrm{kV} / \mathrm{cm}\end{array}$ & $\begin{array}{c}\text { Output } \\
\text { energy, } \mathrm{MeV}\end{array}$ & $\begin{array}{c}\text { Spectrum, } \\
\%\end{array}$ \\
\hline \hline 100 & 19.56 & 0.396 \\
200 & 30.87 & 0.251 \\
300 & 42.24 & 0.231 \\
400 & 53.62 & 0.224 \\
\hline
\end{tabular}

\section{References}

Bondarenko, T.V. et al. (2013) BEAMDULAC-BL code for 3D simulation of electron beam dynamics taking into account beam loading and coulomb field Problems of Atomic Science and Technology. Series: Nuclear Physics Investigations 6 (88) pp. 114-118.

Bulanov, S. et al. (1998) Particle injection into the wave acceleration phase due to nonlinear wake wave breaking Phys. Rev. E 58 R5257.

Feinberg, Ya.B. (1959) Particles acceleration in plasma Sov. Atomic Energy 6 p. 431.

Hogan M.J., et al. (2005) Multi-GeV Energy Gain in a Plasma-Wakefield Accelerator Phys. Rev. Lett. 95 054802-1-4.

Kluchevskaia, Yu.D., Polozov, S.M. (2020) Beam dynamics simulation in a linear accelerator for CERN future circular collider Cybernetics and Physics 9 (2) pp. 98-102.

Polozov, S.M. et al. (2010) Calculation of beam dynamics in traveling-wave accelerators taking account of the current load Atomic Energy 109 (2) pp. 106-112.

Polozov, S.M. (2013)A possible scheme of electron beam bunching in laser plasma accelerators NIM A $\mathbf{7 2 9}$ p. 517.

Polozov, S.M., Rashchikov, V.I. (2018) Longitudinal motion stability of electrons inside the plasma channel of LPWA Cybernetics and Physics 7 (4) pp. 228-232.

Rashchikov, V.I., Polozov, S.M., (2020) Simulation studies on the radiofrequency gun saturated emission $C y$ bernetics and Physics 9 (2) pp. 103-106.

Semenov, T.A. et al. (2019) Electron acceleration up to $\mathrm{MeV}$ level under nonlinear interaction of subterawatt femtosecond laser chirped pulses with Kr clusters Laser Phys. Lett. 16115401.

Shkurinov, A.P. et al. (2017) Interaction of high-intensity femtosecond radiation with gas cluster beam: effect of pulse duration on joint terahertz and $\mathrm{x}$-ray emission IEEE Trans. Terahertz Sci.Technol. 7 pp. 70-79.

Shkurinov, A.P. et al. (2021) Directional terahertz beam generation under interaction of an intense femtosecond 
laser pulse with a cluster jet Journal of the Optical Society of America B 38 (11) 3515.

Tajima, T., Dowson J.M. (1979) Laser Electron Accelerator Phys. Rev. Lett. 43 p. 267.
Umstadter, D., Kim, J.K., Dodd E. (1996) Laser Injection of Ultrashort Electron Pulses into Wakefield Plasma Waves Phys. Rev. Lett. 76 p. 2073. 